ARTICLE

https://doi.org/10.1057/s41599-019-0356-0

\title{
Synchronization: a framework for examining emotional climate in classes
}

Efrat Eilam (1) ${ }^{1 \star}$

\begin{abstract}
This conceptual paper stems from a growing body of research examining the roles and functions of emotional climate (EC) - the collective state of students' shared emotions-in classrooms. It suggests that EC in classrooms can be analyzed as a synchronization phenomenon. Synchronization is a natural phenomenon, in which autonomous objects that produce periodic rhythms adjust these rhythms to one another. The purpose of this article is thus twofold: to position the concept of EC within the broader context of synchronization theory; and to propose a new definition for EC that captures synchronization aspects. This paper first explains the theory of synchronization. It then critically discusses several sociological and biological theories regarding emotions, and the implications of these theories for teaching and learning. It subsequently delves into current conceptualizations of EC, and the descriptors used to characterize EC in classrooms. On the backdrop of this theoretical review, an argument is developed that humans' produced emotions can be viewed as autonomous rhythmic oscillators that have the capacity to synchronize with one another, and that, consequently, EC can be studied as a form of synchronization within an ensemble of oscillators. Finally, a new definition of EC is proposed that integrates essential synchronization features. Overall, this paper suggests that synchronization theory can provide a framework of analysis of EC as a holistic phenomenon, not reduced to discrete emotional events. This approach may contribute to enhancing explanatory and predictive capacities in the study of EC.
\end{abstract}

\footnotetext{
${ }^{1}$ Victoria University, Footscray, VIC 3011, Australia. *email: efrat.eilam@vu.edu.au
} 


\section{Introduction}

his conceptual paper proposes a new framework of analysis for examining the roles and functions of emotional climate (EC) in classrooms. A classroom's EC can be understood as the collective state of students' shared emotions (Bellocchi et al., 2014). Studies suggest that positive classroom emotions enhance both the learning experience and learning outcomes (Bellocchi et al., 2014; Reyes et al., 2012; Trigwell, 2012).

Herein, I propose that EC in classrooms can be considered as an expression of a synchronization phenomenon-a system comprising multiple autonomous individuals that produce periodic rhythms with the capacity to adjust to one another. Additional examples of synchronization phenomena include the conformity of the sounds of organ-pipes, fireflies emitting sequences of light pulses, applauding audiences, and circadian cycles. Though these phenomena might initially seem to have little in common, in fact, they exhibit numerous shared characteristics that enable them to be studied under a unified theory of synchronization (Pikovsky et al., 2001, p. 7). Herein, I propose that this theory can be applied to the study of EC. More specifically, this essay seeks to achieve two main goals: (i) to position the study of EC within the broader context of the naturallyoccurring phenomenon of synchronization; and (ii) to propose a new definition of EC, capable of capturing aspects of synchronization.

The field of study of EC in classrooms has developed substantially over the past decade, advanced primarily by a leading group of researchers in Australia and in the U.S.A. (e.g., Bellocchi et al., 2013; Bellocchi et al., 2014; Davis and Bellocchi, 2018; Rinchen et al., 2016; Ritchie and Beers Newlands 2017; Tobin et al., 2013). Their work, which was conducted primarily within science classrooms, brought EC to the attention of science education researchers, and laid the groundwork for further investigation of EC in classroom settings. Thus far, studies investigating EC in education have tended to adopt an eventoriented analysis approach, using research methodologies such as "auto-ethnographic selfstudies, narratology, interpretive research grounded in hermeneutic phenomenology" (Tobin, 2018, p. 33). These methodologies generally entail using in-depth analysis to unpack various properties of an emotional event (Davis and Bellocchi, 2018; Ritchie, 2018; Ritchie and Beers Newlands, 2017; Tobin, 2018; Tobin et al., 2016).

Building on this body of research, the present article addresses the topic of classroom EC through a different lens. It diverges from prior studies ontologically and epistemologically. Whereas previous studies dissected EC into discrete events, classroom EC is viewed here as a whole-class phenomenon, analogous to a living organ in which the whole is not a cumulative sum of its parts. To understand EC from this perspective, there is a need to develop a framework of analysis that is capable of drawing meanings from the behavior of the organ as whole. The analogy to this claim is that, in the same way that we cannot fully understand how the arm works by in-depth analysis of parts of the arm (for example, ligaments), it is not possible to develop a comprehensive understanding of the effects of EC, or the capacity to measure and predict those effects, without a holistic framework of analysis.

In what follows, I first explain the theory of synchronization. With this theory in mind, I proceed to outline our current understanding of emotions, approaching the topic from both a theoretical-sociological perspective and a biologicalneuroscientific perspective. I then elaborate on the implications of emotions for teaching and learning; critically discuss current definitions and descriptors of EC; and explain the applicability of the theory of synchronization as a framework for researching EC. Finally, I propose a new definition of EC that integrates essential features of synchronization phenomena.

\section{Synchronization}

Synchronization in nature: overview and examples. Synchronization phenomena are abundant throughout nature. Our surroundings are full of oscillating objects that synchronize with one another: clocks; singing crickets; flocks of birds flapping their wings; cooperative behavior of humans and non-human animals, such as insects; the adjustment between heart rate and respiration; the synchronous firing of neurons. All these and many other systems have a common feature: they produce periodic rhythms that have the capacity to adjust to one another (Pikovsky et al., 2001, p. 17).

The word synchronous is derived from Greek. It literally means "occurring in the same time" (Pikovsky et al., 2001, p. 17). Synchronization is a universal phenomenon that "can be understood within a common framework based on modern nonlinear dynamics" (p. 1).

McClintock (1971) was the first to carry out a scientific study of synchronization in a human socio-physiological system. She studied the menstrual periods of 135 undergraduate females living in shared dormitories. She found that menstrual cycles of close friends became increasingly synchronized over the time these individuals spent together in shared accommodations (Strogatz, 1997, p. 2). Particularly interesting was the finding that the greater the extent to which a group of women regarded themselves as "close friends", the stronger the synchronization among them (McClintock, 1971). These findings demonstrate how interpersonal emotional-social processes (close friendships) affect the synchronization of physiological processes (menstrual periods). So far, no mechanisms for explaining the underlying emotive-socio-physiological pathways have been identified.

Definition of key terms. An established set of rules defines what may and what may not be regarded as synchronization (Pikovsky et al., 2001). Key terms relevant to synchronization are defined and explained below, and inclusion and exclusion rules are discussed when applicable.

Synchronization. Synchronization is characterized as "adjustment of rhythms of oscillating objects due to their weak interaction" (Pikovsky et al., 2001, p. 8). This occurs when two or more autonomous objects with different rhythms adjust their rhythms in relation to one another.

This definition implies the following inclusion or exclusion rules:

1. For synchronization to occur, the objects must be oscillators and autonomous systems. The fact that two variables have similar frequencies is not enough to imply that they are synchronous.

2. The objects must be open systems that receive signals from their environment and interact with other objects, such that if these environmental signals are removed, the objects will no longer oscillate in common rhythms. For example, the circadian cycle (the internal biological clock) is influenced by various factors such as exposure to light and temperature. If these signals are removed, the circadian cycle of an organism will deviate from the "normal" 24-h rhythm (Pikovsky et al., 2001, p. 7).

Oscillator. An oscillator is an autonomous, self-sustained system. If perturbed it will attempt to return to its original mode. For example, a clock takes energy from a given source and maintains a steady oscillation of its pendulum until its energy supply ceases. The oscillatory motion is entirely determined by the internal 
parameters of the clock and does not depend on how the pendulum was put into motion (Pikovsky et al., 2001, p. 8).

Rhythm. Autonomous oscillators can exhibit various forms of rhythms such as a sine-like waveform or short pulses. The rhythm is determined by the properties of the system itself. A synchronized object adjusts its rhythm in conformity with the rhythms of other objects (Pikovsky et al., 2001, p. 9). Yet, when two synchronized oscillating systems are separated from each other, they will continue to oscillate in their own rhythms.

Rhythms of oscillating systems can be quantified in various ways. One common means of quantifying a system's rhythm is to count the number of oscillation cycles per time unit, i.e., to measure the frequency of the cyclic oscillations (Pikovsky et al., 2001, p. 9).

Frequency entrainment, phase locking, and phase shift. When synchronization occurs, the frequencies of two or more oscillating objects may be locked. For example, experiments with pendulum clocks have shown that when two independent, non-identical clocks, with different oscillation periods, are placed in proximity to each other, they adjust their rhythms and start to oscillate with a common period. This type of phenomenon is often referred to as "frequency entrainment" (Pikovsky et al., 2001, p. 11).

Phase locking is a term used for describing the onset of a certain relationship between the phases of two synchronized selfsustained oscillators. Phases can be locked such that the oscillators are synchronized to move in the same direction (inphase locking), opposite direction (anti-phase locking), or any variations in between (e.g., moving in the same direction but with a constant frequency lag between the oscillators) (Pikovsky et al., 2001, p. 14).

Mathematically, phase locking does not mean equality of phases but rather phases that keep the same relationships to each other over time. Phases $\varphi 1$ and $\varphi 2$ are referred to as being "n:m locked" if the following inequality holds: $|\mathrm{n} \varphi 1-\mathrm{m} \varphi 2|<$ constant (Pikovsky et al., 2001, p. 23).

When two oscillating objects are synchronized, their motions are not necessarily identical. In our previous example of the synchronized clocks, for example, we might expect to see differences in the motions of the clock pendula due to differences in their initial oscillation periods. The difference in the frequencies of oscillation is termed phase shift. A phase shift will always be present if two systems had initial differences in their oscillation periods. In many cases the differences are subtle and can only be detected with specifically designed instruments. For example, without instrumentational measurements, most people in an applauding crowd will not be able to detect any phase shifts among applauding individuals (Pikovsky et al., 2001).

Synchronization within ensembles of oscillators. Ensembles of oscillators may become synchronized in various ways. Synchronization patterns that are commonly observed in nature and in physical systems include chain, lattice and global (all-to-all) synchronization. In the case of global (all-to-all) synchronization, coupling occurs when there is an ensemble of mutually-coupled oscillators in synchronization. An example of such a selforganizing system is a large population of fireflies flashing in synchrony, or a large audience in a theater applauding in synchrony (Pikovsky et al., 2001, p. 126). An attuned listener in an audience might notice how synchronization is formed through a series of rather fast (several oscillatory periods) transitions from noise to a rhythmic, nearly periodic, applause, culminating in synchronized applause (Pikovsky et al., 2001, p. 19).
In cases of global coupling, each individual responds to the collective behavior (e.g., audience applause or fireflies flashing) of the whole population, rather than to another individual in the group (Strogatz, 1997, p. 3). With regard to the underlying mechanism, Art Winfree (1967) discovered that "synchronization occurs cooperatively, in a manner strikingly reminiscent of a thermodynamic phase transition" (in Strogatz, 1997, p, 3). Winfree (1967) found that when the differences between the individual frequencies are large, the rhythms are incoherent. As the differences between frequencies begin to decrease, at some critical threshold the system spontaneously freezes into synchrony.

Differences between biological oscillators and physical oscillators. Winfree (1967, as cited in Strogatz, 1997) identified two important differences between synchronization among physical objects and synchronization among biological objects. Physical oscillators tend to be self-sustained in the sense that if they are perturbed, they will remember this perturbation forever. In comparison, biological oscillators are selfregulating. They regulate their own amplitude, and, if perturbed, they return to a standard cycle. In addition, physical oscillators can be identical to one another, whereas biological oscillators are never identical, due to genetic variability.

Another difference between physical and biological oscillators relates to measurement. Measuring signals in a biological system tends to be much more complex compared with measuring signals in physical objects (e.g., clocks), because in a biological system it is not possible to manipulate the parameters of the system, such as the coupling strength. The only way to measure interactions between oscillating biological systems is under freerunning conditions (Pikovsky et al., 2001).

Measuring synchronization. Synchronization measurement consists of analyzing the relationships between the phases and frequencies of interacting objects (Rosenblum et al., 2001; Pikovsky et al., 2001, p. 156). The phases may be measured by identifying markers in a time series. For example, in electrocardiography (ECG) measurements, every normal cardio-cycle contains a sharp peak that can be localized in time. The time interval between two such peaks corresponds to one complete cardio-cycle. Therefore, the phase increase during this time interval is exactly $2 \pi$ (Pikovsky et al., 2001, p. 153). The frequency is determined by the number of oscillating cycles per time unit, measured as $2 \pi / T$ (Pikovsky et al., 2001, p. 10).

Because synchronization is not a state but instead a process of adjustment of phases and frequencies, multiple measurements are required to establish the presence of synchronization (Pikovsky et al., 2001, p. 157).

As noted above, the measurement of synchronization in biological systems poses particular challenges due to a lack of access to oscillators' parameters. Even if repeated measurements do reveal relations between oscillators' phases and frequencies, it remains difficult to determine whether these relations are attributable to synchronization or pure coincidence. In light of this limitation, it is important to note that synchronization analysis is based on the assumption that the objects are selfsustained oscillators that either interact or oscillate independently (Pikovsky et al., 2001, p. 168). When attempting to evaluate whether a biological system indeed shows oscillation patterns that can be described by a synchronization model, it is necessary to first confirm this assumption.

To sum up, the discussion above presents the basic assumptions of the theory of synchronization. In later sections, the appropriateness of synchronization models for describing the phenomenon of EC (i.e., as a synchronization of individuals' emotions) will be further addressed. 
Theoretical and biological understanding of emotions. Emotions are composite-dynamic phenomena, which involve interactions between physical properties (such as muscle contractions) and non-physical ones (such as cognitive perceptions), occurring over time and space dimensions (Collins, 2004; Barrett, 2017; Pekrun et al., 2009). Diverse theories of emotions have been developed in various disciplines, adopting a multitude of theoretical lenses. As this work focuses on EC in education, in what follows I discuss and subsequently build on the sociological and neuroscientific theories that are most widely used in this body of research.

As far back as the 1800s, Charles Darwin (1969/1872) in "The Expression of Emotions in Man and Animals" developed three principles that formed the basis of a theory of expression. These principles, whose aim was to explain the occurrence of emotional expression and involuntary gestures, were based on the assumption that emotion, or "state of mind", induces physical expressions.

The first principle, "the principle of serviceable associated habits", suggests that there are associations between states of mind and physical expressions. These associations develop into habitual responses, and they are heritable (p. 28). The first principle recognizes that, though the initial association between a state of mind and a physical expression is formed to provide some service to the organism, this association later turns into a habit that does not necessarily provide a service.

The second principle, "the principle of antithesis", suggests that when opposite states of mind are induced, there is an involuntary tendency to perform movements of opposite nature. Such responses are of no use to the organism (p. 28).

The third principle is "the principle of actions due to the constitution of the nervous system, independently from the first of will, and independently of a certain extent of habit". This principle relates to unmediated direct action by the nervous system (p. 29).

Darwin continues to describe the role of emotions in the development of adaptive behavior and in regulating interactions between individuals who belong to social species. As discussed later in this essay, Barrett (2017) criticized Darwin's ideas about emotions as being essentialist and anti-evolutionist. However, in my view, Darwin's ideas about emotions were extraordinary for his time, in at least three ways: first, they explicitly link mental states with physical bodily expressions; second, they identify the roles that emotions play at both the individual (micro) level and the societal (macro) level, and they create links between the two levels; and third, they acknowledge that non-human animals do exhibit high-order emotions (emotions that are not mere reflex), an idea that was later denounced and only revived again in the late 20th century (Sorabji, 1995).

Building on Darwin's work, William James (1968/1884) and Carl Lange (1885) independently reached a similar conclusion known as the James-Lange theory, according to which a stimulus creates physiological changes that are later interpreted by the cortex as emotion. James (1968/1884) described the following sequence of emotional processes: "the bodily changes follow directly the perception of the exciting fact, and that our feeling of the same changes as they occur is the emotion" (p. 19, as quoted in Scherer, 2000, p. 155). According to this view, emotions are elicited not by the direct experience, but rather by an awareness of physical changes. The perception of emotion is a cognitive interpretation of physiological changes (Scherer, 2000).

Other scholars in the late 19th century, such as John Dewey (1894), continued to grapple with questions related to the universality of emotional expressions and the perceived linear pathways connecting perception, awareness and expression.

Current (21st-century) multi-componential theories of emotion acknowledge that emotions do not behave as a simple linear cause-effect phenomenon; rather, they are better characterized by their complexity (Sander et al., 2005; Scherer, 2004). Scherer (2004) proposed the component-process definition, describing emotions as processes of causallylinked mental elements (appraisal, action tendency, subjective experience) and behavioral elements (physiological reactions, facial and vocal expression). According to this definition, the perception and appraisal of events occur simultaneously rather than sequentially. These in turn trigger changes in all the systems involved in the emotional process.

It is important to note that both the early theories and the more recent theories view cognitive information processing as an essential component of the emotional response, disregarding the widely applied binary view of emotions as separate from cognition. However, assumptions regarding the extent of inseparability vary among different scholars and disciplines.

Sociological theories of emotion. Several sociological perspectives of emotions establish links between the emotions of individuals and social structures (Collins, 2004; Turner, 2007). These perspectives converge to the following ideas: (i) Emotions are hardwired and constitute a hierarchy of primary, secondary and tertiary levels (Turner, 2007). (ii) The expression of emotion influences and is influenced by various social stimuli (Collins, 2004; Turner, 2007). (iii) Though emotion and cognition are highly interconnected, there is a distinct separation between the two (Collins, 2004, p. 107).

Collins' (2004) Interaction Ritual Theory explains emotions as generating and being generated by social rituals, where a ritual is defined as "a mechanism of mutually focused emotion and attention producing a momentarily shared reality, which thereby generates solidarity and symbols of group membership" (p. 7). According to this theory, an individual's perception of reality is constructed by emotional processes in which rituals act as driving forces (Collins, 2004, p. 104). Participation in a ritual gives the individual a special kind of energy, termed emotional energy. Collins (2004) characterizes this energy as: (i) a strong, steady emotion, lasting over a period of time (p. 134) (the "half-life" of emotional energy is suggested to be between a few hours and a few days (p. 150)); (ii) stored in the brain, in attachment to symbols (p. 38); (iii) objectively measurable by outside observers through bodily postures and expressions, interactions, selfreports, and hormonal levels (p. 135).

Despite the centrality of emotions in this theory, the term "emotional energy" remains at the level of a metaphor. Indeed, despite the theory's claim that "the physiology buttresses the sociology" (Collins, 2004, p. 107), recent research in neuroscience suggests that there is no evidence for measurable emotional energy essence (Barrett, 2017). More so, it is questionable whether a metaphor can be empirically observed and measured, in a scientific way. Noble (2008), for example, discusses the limitations of the selfish gene as a metaphor. He provides an opposing metaphor to the selfish gene, and challenges the reader to think of an empirical test that could possibly distinguish between the two diametrically opposing metaphors (p. 11). Noble (2008) concludes that "no metaphors map perfectly to the situation they are describing....the harm is done when we take the metaphors too literally....and interpret them as uniquely correct scientifically" (p. 15).

Turner's (2007) sociological theory of emotion considers emotions as both biological and cultural processes. Cultural norms govern the arousal of particular emotions, which, in turn, affect social encounters and larger social structures. Turner acknowledges tight evolutionary connections between emotions and cognition, yet he does not perceive the two as unified. He states that "it is the interaction between cognitive and emotional 
capacities that makes rationality and memory possible on human scale" (Turner, 2007, p. 37). The theory further suggests that the hierarchical organization of emotions in the brain is derived from the interaction of primary emotions, which create secondary and tertiary levels of emotions (Turner, 2007, p. 6).

Neuroscience-based theories of emotion. Neurobiological research has produced findings consistent with the psychological view that emotions permeate all cognitive functioning, including memory (Damasio, 1994; Davidson and Begley, 2012; Immordino-Yang and Damasio, 2007; Sylwester, 1995) and the various mental processes involved in learning (Rosenfield, 1990). Indeed, it seems that all brain regions are affected by emotions, suggesting that everything that humans do is impacted by emotions (Davidson and Begley, 2012; Barrett, 2017).

Studies examining the neurobiological pathways activated in the presence of emotion have hypothesized that emotional experiences cause certain hormones to be released in the brain, and that these hormones, in turn, influence how information is encoded into structures that elicit memories (Packard and Cahill, 2001, as cited in Titsworth et al., 2010, p. 434). Grossberg (2009) suggested that emotions direct learning through the following pathway: "Incentive motivational learning enables emotions to generate a motivational set that biases the system to process information consistent with that emotion" (p. 1226). This model suggests that emotional concepts can modify how people use previously-learned information to make decisions (Titsworth et al., 2010, p. 434).

Research in neuroscience has given rise to Lisa Feldman Barrett's (2017) Theory of Constructed Emotion. This theory presents emotions as constructs of our brains, rather than builtin. Specifically, Feldman Barrett (2017) suggests that there is no form of inherent emotional fingerprint, or essence of emotion, and that there is no specific location in the brain in which emotions reside (p. 168). Rather, emotions are concepts, which are dependent on a combination of physical properties, the flexibility of the brain to respond to its environment, and the culture and environment in which emotional responses evolve and operate (p. 12). As concepts, emotions serve various purposes. At the level of the individual, they function to: make meanings, prescribe action, and regulate the body budget (pp. 138-139). At the level of society, they function to: (i) form communication, as "two people categorize with concepts in synchrony" (p. 139); and to (ii) create social influence-tools to regulate other people's body budgets" (p. 139). The latter social aspect of emotions provides strong grounds for studying EC as a form of synchronization.

Stemming from this theory is the idea that there is no universality in emotions or in their expressions. Variation, not uniformity, is the norm in regard to emotional bodily responses to the same categories of emotions (p. 23). The theory asserts that emotions are not distinct from cognition and perception (p. 34). Their seeming universality is derived from socially shared concepts (p. 38).

Barrett (2017) further criticizes Darwin (1969/1872), stating that his theory of emotions contradicts his own Theory of Evolution (Darwin, 1859), in that it is essentialist.

This criticism may also be applicable to Collins' (2004) and Turner's (2007) sociological theories of emotions, described above. These theories, too, seem to presuppose that emotions have an essence, meaning an underlying property that causes them to be similar (Barrett, p. 157). Barrett explains that according to the Theory of Evolution, "each species was a conceptual category-a population of unique individuals who vary from one another, with no essence at their core" (p. 159). According to this perception, a concept of the emotion "sadness" is a statistical summary. "It is based on variation, whereas essentialism is based on sameness" (p. 157). Barrett further asserts that "essentialism is remarkably difficult to disprove. Since an essence can be an unobservable property, people are free to believe in essences even when they cannot be found" (p. 162).

Notably, thus far, most classroom studies concerning EC have relied on the sociological theories of emotion reviewed above, and may therefore be vulnerable to similar critiques. In contrast, the approach proposed herein for examining emotional synchronization in EC is firmly rooted in the recent neuroscientific Theory of Constructed Emotions, as will be elaborated in what follows.

The role of emotions in education. The second half of the 20th century saw a gradual shift from the perception of learning as a purely rational process to viewing learning as a complex process in which emotions and cognition are intertwined (Dierking, 2005).

Studying the role of emotions in education poses particular challenges due to the lack of universality in the nature of individuals' emotions, discussed above (Barrett, 2017). Moreover, unlike characteristics commonly attributed to cognition, such as conceptual change, which show some stability over time, emotions tend to undergo fluctuations, making them difficult to capture and measure (Zembylas, 2005). An additional challenge is posed by the gap between the individual's perception of emotions and their neurological expression (Barrett, 2017; Tamietto and de Gelder, 2010; Zembylas, 2005). Emotional stimuli may trigger physiological and behavioral responses without ever being perceived consciously (De Gelder et al., (2001); Tamietto and de Gelder, 2010).

In recent years, research focusing on emotions in science classrooms has undergone expansive development, as reflected in the introduction of new theoretical frameworks, methodologies and methods. Davis and Bellocchi (2018) describe the expansion of theoretical perspectives as a strategy employed by researchers to "wrangle the emotion octopus into a manageable container" (p. 10). Tobin (2018) refers to these emerging research approaches as "multilogical bricolage" (p. 33). This perspective conceptualizes the diverse theoretical and methodological frameworks as complementary to one another, rather than as different and mutually exclusive truth systems. Research grounded in this perspective has a strong ethical basis that focuses on change and educating all participants (Tobin, 2018; Tobin et al., 2017).

A particular application of this approach is in the study of emotional events (Ritchie and Beers Newlands, 2017). An emotional event is "a unit of analysis associated with cultural transformation in the classroom that originated from ruptures in the everyday practices of teachers and/or students to produce salient emotional outcomes" (Ritchie and Beers Newlands, 2017, p. 108). The process of analysis aims to unpack the "temporal, contingent, and transformational properties" of an emotional event (Ritchie and Beers Newlands, 2017, p. 118).

Research to date has been successful in identifying intricate feedback loops and impacts related to the relationships between emotions and learning outcomes. It was suggested that emotions are critically important in relation to other learning aspects such as students' motivation, identity development and learning performance and outcomes (Schutz and Pekrun, 2007 as cited in Pekrun et al., 2011). For example, studies focusing on memory and information retainment have revealed that individuals remember emotional events to a greater extent, and in more detail, than they remember non-emotional events. These observations suggest that emotions contribute to conceptual retention of learning (Immordino-Yang and Damasio, 2007; Kensinger and Schacter, 2008 as cited in King et al., 2015; Nielson and Lorber, 2009; Olafson and Ferraro, 2001). In particular, it was found that heightened emotions are linked to 
long-term memorability of learning activities (Allen, 2010; King et al., 2015).

Other studies relate emotions to specific cognitive performance. For example, it was reported that positive affective states promote creative, flexible, and holistic ways of thinking, whereas negative affective states promote more analytical, detailed, and rigid ways of processing information (Ashby et al., 1999; Fiedler, 2001 as cited in Pekrun et al., 2009, p. 118). Enjoyment of learning tends to be a positive predictor of performance, whereas anger, shame, boredom, and hopelessness tend to be negative predictors (Pekrun et al., 2009, p. 118). In line with these findings, Trigwell et al. (2012) report correlation between positive emotions and learning outcomes such as achievement scores. Reyes et al. (2012) observed a positive association between the extent to which a classroom's EC is positive and the grades that students in the class achieve. Other studies have indicated, however, that both positive and negative emotions are conducive to learning, revealing intricate relationships among the ups and downs of learning processes (Jaber and Hammer, 2016; King et al., 2015; King et al., 2017; Tomas et al., 2016). Bellocchi (2018) investigated disruptive emotional events among 8th-grade students engaged in three types of scientific inquiries: closed non-laboratory; guided laboratory-based; and open laboratory-based. He found that negative emotional events arise when students encounter barriers to the progression of their inquiries, and that some categories of negative emotional events are conducive to learning (e.g., failure during experimental trials), whereas others impede learning (e.g., equipment malfunctions).

Students' self-regulation of emotions has been found to support achievement of desirable learning outcomes (Kershner et al., 2012; Tomas et al., 2016). Additional studies have shown that relaxation techniques such as short deep breathing and mindfulness support regulation of emotions (King et al., 2018; Powietrzyńska and Gangji, 2016), and particularly negative emotions (King et al., 2018). Research findings further highlight the importance of developing teachers' capacity to gauge students' emotions and respond accordingly (Bellocchi and Ritchie, 2015; Davis and Bellocchi, 2018; King et al., 2017; Milne and Rubin, 2011).

Taken together, the studies reviewed above suggest that researchers are just beginning to uncover intricate relationships between emotions and learning. To develop this field further, Linnenbrink-Garcia and Pekrun (2011) call for advances in methodology and approaches to assessing emotions in order to improve understanding regarding the relations between emotions and students' cognitive processes, motivation and academic performance.

Moreover, we observe that current work in this vein largely tends to approach the classroom experience as a collection of discrete units of analysis of emotional events. Thus far, a similar approach has been used in the study of EC as well, as I elaborate below.

\section{Emotional climate}

Definitions. Emotions at the individual level are often governed by social interactions (Zembylas, 2005). Social interactions may be particularly integral to emotion in situations in which multiple people are sharing an experience, as in the case of a classroom in which students are engaged in social encounters. The shared emotions manifest themselves as an EC (Bellocchi et al., 2014).

Thus, EC can be viewed as a form of shared experience, where the term experience refers to the process of "relating information from outside sources to states of consciousness" (Csikszentmihalyi, 2014, p. 211). According to this definition, experience intrinsically includes both external signals and the individual's responses to them.

Over the years, researchers have proposed various refinements for the general concept of EC as a manifestation of shared emotions. De Rivera (1992), for example, distinguished the concept of EC from that of emotional atmosphere. Specifically, according to his definitions, emotional atmosphere refers to "the collective behavior that a group or society may manifest when it is focused on a common event" (p. 2), whereas EC refers to "the emotional relationships between members of the society" (p. 2). Thus, these two early definitions separate between the behavior of the group and the relationships within it.

Barbalet (1995) merged the two aspects and broadened the definition of EC by including the context and impact of the EC formation process. Specifically, he defined EC as "sets of emotions or feelings which are not only shared by groups of individuals implicated in common social structures and processes but which are also significant in the formation and maintenance of political and social identities and collective behaviour" (p. 23).

de Rivera and Páez (2007) later refined the earlier definition to include not only group relations but also the context in which the relations are formed. They described EC as the "predominant collective emotions generated through the social interaction of a group's members in a particular milieu" (p. 235).

In the context of the classroom, Evans et al. (2009) conceptualized EC as primarily "the interchange of feelings between teacher and student" that takes place during class ( $\mathrm{p}$. 140). Oakley (2018) highlighted the momentary aspect of EC.

Páez and Rimé (2014) related Durkheim's (1912) notion of emotional communion to the concept of EC. They noted that "every affect-loaded event brings people together and elicits a process of emotional communion or perceived emotional synchrony, composed by emotional contagion and synchrony with others, that supports fusion of identity" (p. 3). This process of emotional communion, in turn, develops into EC through the following process:

"As regards emotional communion or perceived emotional synchrony, collective flow involves the transmission of non-verbal and verbal emotions and moods. This shared emotional arousal causes the creation of a common emotional climate" (p. 5). Páez and Rimé (2014) referred to an earlier study by de Rivera and Páez (2007), showing a linear relation between emotional communion and EC, when measured as variables. Specifically, higher levels of emotional communion are associated with stronger EC (de Rivera and Páez, 2007).

Bellocchi et al. (2014), drawing on Turner's (2007) Sociological Theory of Emotions and on Collins' (2004) Interaction Ritual Theory, described EC as a heightened group experience leading to the development of shared ideas that are representative of the group's interactions. They described the processes by which EC emerges as follows:

Emotional climate is produced during social encounters from which participants develop solidarity or group belongingness, through rhythmic coordination of gesture and speech, mutual focus of attention, production of collective effervescence through group laughter and emotional attunement, and emotional energy. As a ritual outcome, collective effervescence is a state of heightened group experience whereby the group shares the same emotions (e.g., joy) and ideas. Through this process, shared ideas become symbols representing the group's interactions. The heightened emotional state experienced in forming these shared ideas flows on to the emotional energy experienced by individuals. (Bellocchi et al., 2014, p. 1304)

The above description of EC refers to the context of a classroom setting. When examining the applicability of these 
characteristics to other social contexts, the following questions may arise:

- Does a state of EC necessitate the presence of complex feelings such as solidarity and sense of belonging?

- To what extent do the processes described as "rhythmic coordination of gesture and speech, mutual focus of attention, production of collective effervescence ... and emotional attunement, and emotional energy" (p. 1304) constitute essential features in the development of EC?

- To what extent is the outcome state described as "a ritual outcome...a state of heightened group experience whereby the group shares the same emotions....and ideas" (p. 1304) a consistent feature that characterizes the outcome state of EC?

- Does EC necessitate the formation of shared ideas?

To address these questions and refine our understanding and definition of EC, it is useful to consider other situations in which shared emotions develop in relation to a shared experience. Examples include an audience at a concert clapping their hands rhythmically when applauding the performers, or a group of physically remote people sharing emotions of grief (e.g., through online or various media channels) in response to an unexpected and subjectively perceived negative experience, such as a terrorist attack, the unexpected election of a disliked candidate, or the sudden death of a beloved public figure. These examples entail the emergence of shared emotions that may be regarded as states of EC, yet neither example presents the full scope of processes and outcomes described by Bellocchi et al. (2014).

In the example of audience applause, although we can expect EC to emerge through the shared experience, it is less reasonable to expect the development of solidarity, a sense of belonging, or shared ideas. It is not even clear to what extent it is possible to identify the presence of "mutual focus of attention" (Bellocchi et al., 2014, p. 1304) -in which people are focusing on each other mutually (one-to-one) - or, alternatively, diffused attention, in which attention is not given to any particular individual, but rather to the group (audience) as a whole (one-to-all).

In the experience of shared public grief, the EC is shared by a group of people who are having the same experience, yet may be geographically remote from one another. In this example, some of the above descriptors of processes leading to the development of EC (such as "rhythmic coordination of gesture") are unlikely to occur. Similarly, though the grieving individuals share the emotion of grief, they might not necessarily develop shared "ideas".

Accordingly, shared ideas do not become "symbols representing the group's interactions". Indeed, there are no direct physical interactions (though media-mediated interactions do occur).

Both examples of the applauding audience and public grief represent settings in which EC may arise, and in which some of Bellocchi et al.'s (2014) descriptors are not necessarily present.

Thus, it seems that, compared with Bellochi et al.'s (2014) highly specific definition, the definitions offered by Barbalet (1995) and by de Rivera and Páez (2007) are more appropriate as generally inclusive definitions of EC. Both definitions achieve inclusivity through their focus on outputs and outcomes, without making assumptions regarding the processes. Accordingly, these definitions fit more closely with the assumptions of synchronization theory, as will be elaborated in the section on "Studying EC in the Context of Synchronization".

Research and measurement of classroom emotional climate. Numerous studies examining classroom EC have focused on the connection between EC and the quality of classroom experiences (Bellocchi et al., 2014; James, 1971; Pekrun and Stephens, 2010; Trigwell, 2012).
Some studies, working within the framework of Interaction Ritual Theory (Collins, 2004), have attempted to measure EC (e.g., Bellocchi et al., 2013; Rinchen et al., 2016). Recall that according to this theory, EC is, in part, a function of emotional energy, generated by participation in rituals. Accordingly, studies grounded in this theory used variables of EC and emotional energy, which were assumed to take either positive or negative valences, which could change congruently (Bellocchi et al., 2014; Bellocchi et al., 2013; Oakley, 2018; Olitsky, 2013; Rinchen et al., 2016; Tobin et al., 2013). To obtain the valence of EC, researchers asked participants to rate their perceptions of the class's EC on a Likert scale at given time intervals (Bellocchi et al., 2013; Oakley, 2018; Tobin et al, 2013). Ratings of 4 and 5 were considered to reflect positive valence, and ratings of 1 and 2 were considered to reflect negative valence (Bellocchi et al., 2013, p. 538; Rinchen et al., 2016, p. 612; Tobin et al., 2013, p. 76). Participants' responses were then averaged to determine the overall EC.

This method of obtaining a measure of EC raises a few methodological questions: First, it does not measure EC directly; nor does it measure the process by which EC is generated. Rather, it indirectly assesses each individual's subjective perceptions of the EC of the class as a whole. Notably, this measurement approach is not only indirect but also of questionable validity: It is not clear how an individual may be capable of rating an abstract sum of a group's emotions. Did participants indeed rate the group's EC, or was it actually their own individual emotions that they were rating? No validation test was carried out to examine whether the measurement actually captured what it was meant to measure.

To my knowledge, no studies thus far have directly measured the ways in which individual emotions interact spatially and temporally over a class period to create EC, as an outcome effect. It is still an open question as to how an assembly of different emotions converge into a more-or-less unified form of classroom emotion. Moreover, no theoretical framework has yet been proposed that provides a means for measuring the EC phenomenon itself, rather than measuring mere perceptions of EC.

Studying emotional climate in the context of synchronization. I propose that humans' constructed emotions can be viewed as oscillators that have the capacity to synchronize with one another, and that it is therefore possible to study EC as a form of synchronization in an ensemble of oscillators. I further propose that this perspective can substantially enrich EC research, potentially producing novel insights with regard to established phenomena.

Applicability of synchronization theory to the study of shared human emotion. Humans can act as autonomous oscillating objects that synchronize on the group level, as exhibited in the examples of the applauding audience and the synchronization of menstrual periods among women friends (Pikovsky et al., 2001). In addition, humans contain and regulate internal oscillating subsystems, as in the case of the synchronization of heart pacemaker cells (Torre, 1976) or the circadian cycle (Pikovsky et al., 2001).

Humans, if viewed as oscillating objects in an emotional context, are autonomous, open and self-regulated systems. When two individuals are separate from each other, they continue to generate independent emotions. Humans' emotions are highly dependent on environmental signaling. Indeed, this dependence is inherent to the various definitions of emotions discussed above. Specifically, the psychological, sociological and biological perspectives all acknowledge that emotions are responsive to signals from both the internal and external environment. Likewise, the relationship between emotion and the environment is captured in 
Turner's (2007) proposition that "from a cognitive perspective, emotions are conscious feelings about self and objects in the environment" (p. 2). Thus, as typical of biological systems, humans are non-identical, and they are self-regulating systems. Emotions are regulated both internally and externally, as exemplified in the concept of emotional work (Titsworth et al., 2010).

In order for a synchronization model to apply to human emotion, the oscillation of emotion must be characterized by rhythms. Here, too, human systems fulfill that criterion. In general, all aspects of human physiology exhibit rhythms. The most apparent rhythms are those of the 24 -h cycles of wake and sleep; times of peak hunger; and levels of cortisol, a stress hormone, typically exhibiting a steep decline throughout the day and is regenerated during sleep (Kirschbaum and Hellhammer, 1989, in Stone et al., 2006). The various rhythms exhibited by humans are governed by external and internal physiological signals, or environmental cues (Stone et al., 2006).

Human rhythms are not restricted only to physiological patterns. Various studies have shown the existence of emotional diurnal cycles (Monk et al., 1985; Wood et al., 1992). Stone et al. (2006) identified rhythmic cycles consisting of positive and negative emotions. Positive emotions reached their peaks at noon and at about 7 p.m.; in the latter case, the higher levels of positive emotions persisted throughout the evening. Negative emotions reached their peaks at 10 a.m. and at 4 or 5 p.m.

A more recent study used functional-MRI scanning to show how networks of brain areas "tick together" in individuals who are responding to the same emotional event (Nummenmaaa et al., 2012, p. 9599). The study suggested that observation of "scenes resembling everyday life-is associated with inter-subject synchronization of brain circuitries related to emotional, attentional, and mentalizing processes". These processes were found to depend on the valence and intensity of the emotions (Nummenmaaa et al., 2012, p. 9601).

Taken together, the ideas outlined above support the premise that the theory of synchronization is applicable to the study of shared emotion, or EC. In the context of classroom emotions, the confined space and time provide ideal conditions for measuring the processes by which the emotions of students become synchronized spatially and temporally, and how these in turn affect their learning.

Benefits of considering emotional climate as a form of synchronization. A key benefit of the use of synchronization theory as a framework for analysis of EC within education settings, is that this perspective enables EC to be conceptualized as a holistic phenomenon, not reduced to discrete emotional events. Thus, using the theory of synchronization as a framework for researching EC could both refine and increase the explanatory capacity and the predictive capacity of research in the field. Such contributions might be achieved through measurement of variables that are inherent to synchronization theory and that have not yet been systematically incorporated into research of EC, including timing, phase and frequency of EC; the ways in which EC spreads, rises and falls over time periods such as a lesson or a semester; and the environmental signals and triggers that influence the development of an EC.

Additional benefits of using a synchronization framework include the capacity to obtain quantitative measurements of various EC phenomena that have thus far been described only qualitatively. For example, Bellocchi et al. (2013) and Bellocchi et al. (2014) reported that positive EC emerged when the classroom interaction was dominated by dialogues rather than monologues. This observation may suggest that EC is influenced by the quantity of interactions between individuals. This proposition could be examined, and the nature of the influence quantified, by formulating a synchronization model describing $\mathrm{EC}$ as a function of the number of interpersonal interactions.

A synchronization framework may also be useful for elucidating processes of emotional contagion, a phenomenon in which individual emotions spread among members of a group to become a collective emotion. The concept of emotional contagion stems from the work of Durkheim (1912) and is currently used widely in social psychology (e.g., Collins 2004; Páez and Rimé, 2014), as well as in emotional research in the context of science education (e.g., Bellocchi et al., 2014). Bellocchi et al., (2014) explained that "emotional contagion serves to explain how the emotions experienced by individuals can lead to the development of a collective climate and emotional attunement" (p. 1305). Thus far, studies evaluating EC-using participants' self-reported perceptions of the EC in their environment-have measured what may be regarded as the perceived "attunement" aspect of EC (Bellocchi et al., 2013; Rinchen et al., 2016; Tobin et al., 2013). However, to my knowledge, no measurements have been developed to capture the "contagion" aspect of EC.

The theory of synchronization may offer means of measuring both emotional contagion (the process by which individual emotions interact) and attunement (the EC outcome state), by measuring the temporal and spatial processes of frequency adjustments related to emotions. Thus, instead of measuring the perceived valence of EC, it becomes possible to characterize attunement on the basis of its rhythm, frequency entrainment, and phase locking. Notably, synchronization measurements can only take absolute values, i.e., there is no negative synchronization.

Application of a synchronization framework to the study of EC may also enable researchers to observe analogies between EC and other synchronization phenomena. For example, it is possible that some of the global coupling processes suggested to be at work in the synchronization of applauding audiences (Pikovsky et al., 2001) also take place during the development of EC among interacting individuals in the classroom. The group of students and the teacher could be viewed as an ensemble of non-identical oscillators (p. 126), each with its own emotional frequency. As the classroom interactions increase, more and more oscillators tend to be "like their neighbors". The emotional frequencies may become distributed over some range; this distribution has a maximum around the most probable frequency, which could be a positive emotion. This process develops, and eventually almost all the individuals join the majority and oscillate in synchrony, giving rise to EC (Pikovsky et al., 2001, p. 128). Synchronization theory provides means for measuring this spatial and temporal

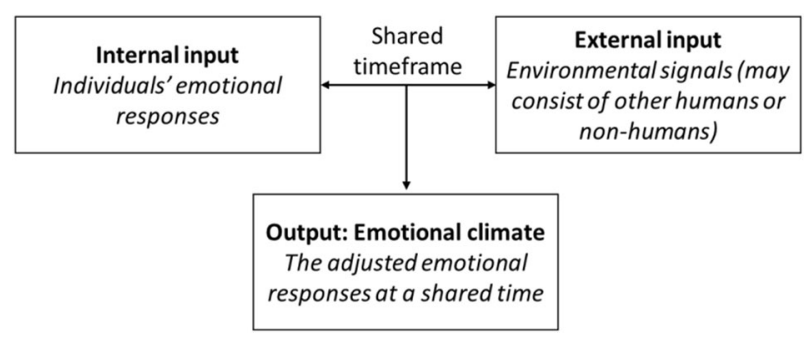

Fig. 1 Interactional processes leading to the development of emotional climate: a representation of the processes contributing to EC development that takes into account a synchronization framework. In this context, an emotional climate state arises as an outcome of individuals' adjusted responses to a common set of environmental signals in a shared timeframe. The signals may consist of inputs by other individuals, a one-off event, a process, or a social structure 
spread of emotions, for example, by applying the mean field theory (Strogatz, 1997).

In sum, the use of synchronization theory as a framework for studying EC seems both appropriate and potentially highly beneficial for the advancement of our understanding of the development of shared emotions. Yet, to be able to adopt this theoretical perspective, we must first revise existing definitions of EC, and particularly the definition proposed by Bellocchi et al. (2014), which is commonly adopted in studies of EC.

A new definition of emotional climate. Considering EC in the context of synchronization gives rise to the following redefinition: Emotional climate is the adjustment of emotional responses among individuals as an outcome of their interactions with a set of environmental signals over a shared period of time.

Figure 1 provides a visual representation of the processes contributing to EC, according to this definition.

The definition captures the following attributes characterizing both EC and synchronization:

- An EC state arises as an outcome of individuals' adjusted responses to a set of environmental signals. The signals may consist of inputs by other individuals, a oneoff event, a process, or a social structure.

- For EC to occur, two conditions must be met: (a) The adjustment between the emotional responses of the various implicated individuals must occur at a shared time; and (b) this emotional adjustment must occur in response to the same set of environmental signals. These stipulations imply that if a group of individuals share emotions developed in response to environmental signals occurring at different times, or different environmental signals occurring at the same time, this sharing cannot be regarded as an EC state.

- An EC state does not necessitate physical face-to-face contact. As long as individuals adjust their responses to the same environmental signals in the same timeframe, the state may be regarded as EC.

- The adjusted emotional responses in an EC state do not necessitate equal emotions or equal frequency of adjustment across the individuals in the system. They do need to demonstrate a consistent relationship over the timeframe of the EC state.

- The process in which individuals adjust their emotional responses must occur at an earlier time point than the emergence of the state of EC, which flows from it.

- EC is confined in time and space (virtual or physical), as defined by the specific environmental signals that give rise to this state. As soon as these signals are removed, the EC state ceases to exist.

The proposed definition captures essential features of synchronization. Accordingly, it opens opportunities for a range of educational research, aiming to gain deeper understanding regarding the relationship between EC and learning. Under the framework of this definition various research pathways may be imagined. These may include, the examination of various environmental signals in relation to learning. We can ask for example: What types of synchronization patterns are formed in outdoor leaning (e.g., excursions, field-trips), as compared to indoor learning, and how these types of synchronization affect learning and retention? Other studies may look at various environmental signals within the classroom itself in relation to their timing and administration. Overall, this definition captures both aspects of classroom EC: the contagion (the input stages) and the attunement (the output stages), and allows their study within the framework of synchronization.

\section{Conclusions}

Synchronization theory has the potential to advance our understanding of the ways in which students' emotions interact within classes, and the impact of these interactions on the learning experience. Ultimately, the usefulness of synchronization theory in EC research will be determined by its capacity to provide deeper understanding regarding the bi-directional relationships between classroom EC as a synchronous behavior and students' individual learning processes.

The positioning of EC as a measurable synchronization output gives rise to several interesting research questions. For example: What are the impacts of various frequencies and phases measured in EC on learning retention? How do various environmental signals in the form of learning activities impact the frequencyrhythms expressed in the EC state? How does the timing of appearance of EC within the learning sequence impact learning and retention (e.g., would it be more effective to elicit a specific EC at the beginning, the middle or the end of a studied unit)? What types of emotions are likely to contribute to the development of ECs of particular characteristics?

Synchronization theory may also facilitate the development of explanations, from an EC perspective, regarding the role of emotions in teaching and learning. In regard to science classes specifically, several studies have highlighted some of the knowledge gaps in understanding the role of emotions in science education. These gaps include: (a) the science activities that evoke specific emotions, and the impact of these emotions on students' memory of the experience (King et al., 2015, p. 1887); (b) the relations between emotions and the gender gap in the sciences (Alsop, 2005); and (c) the link between discrete achievement emotions and academic performance (Pekrun et al., 2009).

Additional unknowns relate to the education of preservice teachers in science, and include the following: (a) What is the role of emotions in becoming and being science teachers (Bellocchi et al., 2014, p. 1306) (b) How do the emotions experienced by preservice teachers relate to their understanding of scientific concepts and science teaching (Alsop, 2005, p.126) (c) What is the relationship between emotions, emotional climate and the quality of science teacher education in university classes (Bellocchi et al., 2014, p. 1301) While much of the research conducted in recent years has focused on the science classroom, most of these questions are applicable to education at large.

Most importantly, the theory of synchronization may open new possibilities for viewing and studying the classroom EC as a "living organ" with its own internal dynamics, rather than as an assembly of discrete individuals involved in the learning experience.

Received: 6 May 2019; Accepted: 28 October 2019; Published online: 19 November 2019

\section{References}

Allen M (2010) Learner error, affectual stimulation, and conceptual change. J Res Sci Teach 47:151-173

Alsop S (2005) Bridging the Cartesian divide: science education and affect. In Alsop S (ed) Beyond Cartesian Dualism: encountering affect in the teaching and learning of science. Springer, Dordrecht, pp. 3-15

Ashby FG, Isen AM, Turken AU (1999) A neuropsychological theory of positive affect and its influence on cognition. Psychological Rev 106:529-550

Barbalet JM (1995) Climates of fear and socio-political change. J Theory Soc Behav 25:15-33

Barrett LF (2017) How emotions are made: the secret life of the brain. Houghton Mifflin Harcourt, Boston 
Bellocchi A (2018) Chapter 5: Negative emotional events during science inquiry. In: Ritchie SM, Tobin K (eds) Eventful Learning. Learner Emotions. Brill Sense, Leiden/Boston, pp. 9-30

Bellocchi A, Ritchie SM, Tobin K, Sandhu M, Sandhu S (2013) Exploring emotional climate in pre-service science teacher education. Cultural Stud Sci Educ 8:529-552. https://doi.org/10.1007/s11422-013-9526-3

Bellocchi A, Ritchie SM, Tobin K, King D, Sandhu M, Henderson S (2014) Emotional climate and high quality learning experiences in science teacher education. J Res Sci Teach 51(10):1301-1325. https://doi.org/10.1002/ tea. 21170

Bellocchi A, Ritchie SM (2015) “I was proud of myself that I didn't give up and I did it": experiences of pride and triumph in learning science. Sci Educ 99:638-668. https://doi.org/10.1002/sce.21159

Collins R (2004) Interaction ritual chains. Princeton University Press, Princeton

Csikszentmihalyi M (2014) Flow and the foundations of positive psychology. The collected works of Mihaly Csikszentmihalyi. Springer, Dordrecht Heidelberg, New York, London

Damasio AR (1994) Descartes' error: emotion, reasons, and the human brain. Avon Books, New York

Darwin C (1859) On the origin of species by means of natural selection, or preservation of favoured races in the struggle for life. John Murray, London

Darwin C (1969) The expression of the emotions in man and animals. University of Chicago Press, Chicago. (Original work published 1872)

Davidson RJ, Begley S (2012) The emotional life of your brain. How its unique patterns affect the way you think, feel, and live-and how you can change them. Hudson Street Press, New York

Davis JP, Bellocchi A (2018) Chapter 2: Emotions in learning science. In: Ritchie S, Tobin K (eds) Eventful learning. Learner emotions. Brill Sense, Leiden/Boston, pp. 9-30

De Gelder B, de Haan E, Heywood C (2001) Out of mind. Varieties of unconscious processes. Oxford University Press, Oxford

De Rivera J (1992) Emotional climate: Social structure and emotional dynamics. In: Strongman KT (ed) International review of studies on emotions, Vol. 2. John Wiley \& Sons Ltd., London, pp. 197-218

de Rivera JH, Páez D (2007) Emotional climate, human security, and cultures of peace. J Soc Issues 63(2):233-253

Dewey J (1894) The theory of emotions: emotional attitudes. Psychological Rev 1 (6):553-569

Dierking LD (2005) Museums, affect, and cognition: the view from another window. In: Alsop S (ed) Beyond Cartesian Dualism: encountering affect in the teaching and learning of science. Springer, Dordrecht, pp. 111-122

Durkheim E (1912) Les formes élémentaires de la vie religieuse. Alcan, Paris

Evans IM, Harvey ST, Buckley L, Yan E (2009) Differentiating classroom climate concepts: Academic, management, and emotional environments. NZ J Soc Sci Online 4(2):131-146. https://doi.org/10.1080/1177083×.2009.9522449

Fiedler K (2001) Affective states trigger processes of assimilation and accommodation. In: Martin LL, Clore GL (eds) Theories of mood and cognition: a user's guidebook. Erlbaum, Mahwah, pp. 85-98

Grossberg S (2009) Cortical and subcortical predictive dynamics and learning during perception, cognition, emotion and action. Philos Trans R Soc 364:1223-1234. https://doi.org/10.1098/rstb.2008.0307

Immordino-Yang M, Damasio A (2007) We feel, therefore we learn: the relevance of affective and social neuroscience to education. Mind, Brain Educ 1(1):3-10

Jaber LZ, Hammer D (2016) Learning to feel like a scientist. Sci Educ 100 (2):189-220. https://doi.org/10.1002/sce.21202

James H (1971) Attitude and attitude change: its influence upon teaching behavior. J Res Sci Teach 8:351-355

James W (1968) What is an emotion? Mind 9:188-205. Original work published 1884

Kensinger E, Schacter D (2008) Memory and emotion. In: Lewis M, HavilandJones J, Feldman Barrett L (eds) Handbook of emotions. Guilford Press, New York, pp. 601-617

Kershner R, Warwick P, Mercer N, Kleine Staarman J (2012) Primary children's management of themselves and others in collaborative group work: 'Sometimes it takes patience...' Education 3-13 42(2):201-216. https://doi.org/ 10.1080/03004279.2012.670255

King D, Ritchie S, Sandhu M, Henderson H, Boland B (2017) Temporality of emotion: antecedent and successive variants of frustration when learning chemistry. Sci Educ 101(4):639-672. https://doi.org/10.1002/sce.21277

King D, Ritchie S, Sandhu M, Henderson H (2015) Emotionally intense science activities. Int J Sci Educ 37(12):1886-1914. https://doi.org/10.1080/ 09500693.2015.1055850

King D, Sandhu M, Henderson S, Ritchie SM (2018) Chapter 10: Managing emotions. Outcomes of a breathing intervention in Year 10 science. In: Ritchie SM, Tobin K (eds) Eventful learning learner emotions, Brill Sense, Leiden/Boston, pp. 193-216

Kirschbaum C, Hellhammer D (1989) Salivary cortisol in psychobiological research: an overview. 1. Neuropsychobiology 22:150-169
Lange CG (1885) Om sindsbevägelser. Copenhagen

Linnenbrink-Garcia L, Pekrun R (2011) Students' emotions and academic engagement: Introduction to the special issue. Contemp Educ Psychol 36 (Special issue):1-3. https://doi.org/10.1016/j.cedpsych.2010.11.004

McClintock MK (1971) Menstrual synchrony and suppression. Nature 229:244-245

Milne C, Rubin K (2011) Embodying emotions: making transactions explicit in science learning contexts. Cultural Stud Sci Educ 6:625-633. https://doi.org/ 10.1007/s11422-011-9354-2

Monk T, Fookson J, Moline M, Pollack C (1985) Diurnal variation in mood and performance in a time-isolated environment. Chronobiol Int 2:185-193

Nielson KA, Lorber W (2009) Enhanced post-learning memory consolidation is influenced by arousal predisposition and emotion regulation but not by stimulus valence or arousal. Neurobiol Learn Mem 92:70-79

Noble D (2008) The music of life. Biology beyond genes. Oxford University Press, Oxford

Nummenmaaa L, Glereanb E, Viinikainenb M, Jääskeläinenb IP, Haria H, Sams M (2012) Emotions promote social interaction by synchronizing brain activity across individuals. PNAS 109(24):9599-9604. https://doi.org/10.1073/ pnas. 1206095109

Oakley JL (2018) Chapter 9: Cogenerative dialogue and classroom emotional climate. Engaging with difficult students. In: Ritchie SM, Tobin K (eds) Eventful learning. Learner emotions. Brill Sense, Leiden/Boston, pp. 171-192

Olafson KM, Ferraro FR (2001) Effects of emotional state on lexical decision performance. Brain Cognition 45:15-20

Olitsky S (2013) We teach as we are taught: exploring the potential for emotional climate to enhance elementary science preservice teacher education. Cultural Stud Sci Educ 8(3):561-570. https://doi.org/10.1007/s11422-013-9530-7

Packard MG, Cahill L (2001) Affective modulation of multiple memory systems. Curr Opin Neurobiol 11:752-756

Páez D, Rimé B (2014) Collective emotional gatherings: their impact upon identity fusion, shared beliefs, and social integration. In: Von Scheve C, Salmela M (eds) Collective emotions. Oxford University Press, Oxford

Pekrun R, Stephens EJ (2010) Achievement emotions in higher education. In: Smart JC (ed) Higher education: handbook of theory and research, 25. Springer, Dordrecht, pp. 257-306

Pekrun R, Elliot AJ, Maier MA (2009) Achievement goals and achievement emotions: testing a model of their joint relations with academic performance. J Educ Psychol 101(1):115-135. https://doi.org/10.1037/a0013383

Pekrun R, Goetz T, Frenzel AC, Barchfeld P, Perry RP (2011) Measuring emotions in students' learning and performance: the achievement emotions questionnaire. Contemp Educ Psychol 36(Special issue):36-48. https://doi.org/ 10.1016/j.cedpsych.2010.10.002

Pikovsky A, Rosenblum M, Kurths J (2001) Synchronization: a universal concept in nonlinear sciences. Cambridge Nonlinear Science Series 12. Cambridge University Press, Cambridge, New York, Melbourne, Madrid, Cape Town, Singapore, São Paulo

Powietrzyńska M, Gangji AKH (2016) "I understand why people need to ease their emotions": Exploring mindfulness and emotions in a conceptual physics classroom of an elementary teacher education program. Cultural Stud Sci Educ 11(3):693-712. https://doi.org/10.1007/s11422-016-9772-2

Reyes MR, Brackett MA, Rivers SE, White M, Salovey P (2012) Classroom emotional climate, student engagement, and academic achievement. J Educ Psychol 104(3):700-712. https://doi.org/10.1037/a0027268

Rinchen S, Ritchie SM, Bellocchi A (2016) Emotional climate of a pre-service science teacher education class in Bhutan. Cultural Stud Sci Educ 11 (3):603-628. https://doi.org/10.1007/s11422-014-9658-0

Ritchie SM (2018) Events in learning science. In: Ritchie SM, Tobin K (eds) Eventful learning. Learner emotions. Brill Sense, Leiden/Boston, pp. 1-7

Ritchie SM, Beers Newlands J (2017) Chapter 6: Emotional events in learning science. In: Bellocchi A, Quigley C, Otrel-Cass K (eds) Exploring emotions, aesthetics and wellbeing in science education research. Springer International Publishing, Basel, pp. 107-120

Rosenblum M, Pikovsky A, Kurths J, Schafer C, Tass PA (2001) Phase synchronization: from theory to data analysis. In: Moss F, Gielen S, Hoff AJ (eds) Handbook of biological physics, Vol. 4, Neuro-informatics. Elsevier Science, New York, pp. 279-321

Rosenfield I (1990) The invention of memory. Basic Books, New York

Sander D, Grandjean D, Pourtois G, Schwartz S, Seghier ML, Scherer KR, Vuilleumier P (2005) Emotion and attention interactions in social cognition: brain regions involved in processing anger prosody. NeuroImage 28:848-858

Scherer KR (2000) Emotions as episodes of subsystems synchronization driven by nonlinear appraisal processes. In: Granic I, Lewis MD (eds) Emotion, development, and self organization: dynamic systems approaches to emotional development. Cambridge University Press, New York, pp. 70-99

Scherer KR (2004) Feelings integrate the central representation of appraisal-driven response organization in emotion. In: Manstead ASR, Frijda NH, Fischer AH 
(eds) Feelings and emotions: the Amsterdam symposium. Cambridge University Press, Cambridge, pp. 136-157

Schutz PA, Pekrun R (eds) (2007) Emotion in education. Academic Press, San Diego

Sorabji R (1995) Animal minds and human morals. The origins of the western debate. Cornell University Press, Ithaca

Stone AA, Schwartz JE, Schwartz N, Schkade D, Krueger A, Kahneman D (2006) A population approach to the study of emotion: diurnal rhythms of a working day examined with the day reconstruction method. Emotion 6(1):139-149. https://doi.org/10.1037/1528-3542.6.1.139

Strogatz SH (1997) Spontaneous synchronization in nature. In: Proceedings of the 1997 International Frequency Control Symposium. IEEE, Orlando

Sylwester R (1995) In celebration of neurons. Association for Supervision and Curriculum Development, Alexandria

Tamietto M, de Gelder B (2010) Neural bases of the non-conscious perception of emotional signals. Nat Rev Neurosci 11:697-709

Titsworth S, Quinlan MM, Mazer JP (2010) Emotion in teaching and learning: development and validation of the classroom emotions scale. Commun Educ 59(4):431-452. https://doi.org/10.1080/03634521003746156

Tobin K (2018) Methodological bricolage. In: Ritchie SM, Tobin K (eds) Eventful learning. Learner emotions. Brill Sense, Leiden/Boston, pp. 31-56

Tobin K, Alexakos K, Malyukova A, Gangji AH (2017) Chapter 12: Jin Shin Jyutsu and ameliorating emotion, enhancing mindfulness, and sustaining productive learning environments. In: Bellocchi A, Quigley C, Otrel-Cass K (eds) Exploring emotions, aesthetics and wellbeing in science education research. Springer International Publishing, Basel, pp. 221-248

Tobin K, King D, Hendersen S, Bellocchi A, Ritchie SM (2016) Expression of emotions and physiological changes during teaching. Cultural Stud Sci Educ 11:669-692. https://doi.org/10.1007/s11422-016-9778-9

Tobin K, Ritchie SM, Oakley J, Mergard V, Hudson P (2013) Relationships between EC and the fluency of classroom interactions. Learn Environ Res 16 (1):71-89. https://doi.org/10.1007/s10984-013-9125-y

Tomas L, Rigano D, Ritchie SM (2016) Students' regulation of their emotions in a science classroom. J Res Sci Teach 53(2):234-260. https://doi.org/10.1002/ tea. 21304

Torre V (1976) A theory of synchronization of heart pace-maker cells. J Theor Biol 61(1):55-71

Trigwell K (2012) Relations between teachers' emotions in teaching and their approaches to teaching in higher education. Instructional Sci 40:607-621

Trigwell K, Ellis RA, Han F (2012) Relations between students' approaches to learning, experienced emotions and outcomes of learning. Stud High Educ 37 (7):811-824. https://doi.org/10.1080/03075079.2010.549220
Turner JH (2007) Human emotions: a sociological theory. Routledge, London Winfree AT (1967) Biological rhythms and the behavior of populations of coupled oscillators. J Theor Biol 16:15-42

Wood C, Magnello M, Sharpe M (1992) Fluctuations in perceived energy and mood among patients with chronic fatigue syndrome. J R Soc Med 85:195-198

Zembylas M (2005) Emotions and science teaching: present research and future agendas. In: Alsop S (ed) Beyond Cartesian Dualism: encountering affect in the teaching and learning of science. Springer, Dordrecht, pp. 123-132

\section{Competing interests}

The author declares no competing interests.

\section{Additional information}

Correspondence and requests for materials should be addressed to E.E.

Reprints and permission information is available at http://www.nature.com/reprints

Publisher's note Springer Nature remains neutral with regard to jurisdictional claims in published maps and institutional affiliations.

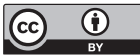

Open Access This article is licensed under a Creative Commons Attribution 4.0 International License, which permits use, sharing, adaptation, distribution and reproduction in any medium or format, as long as you give appropriate credit to the original author(s) and the source, provide a link to the Creative Commons license, and indicate if changes were made. The images or other third party material in this article are included in the article's Creative Commons license, unless indicated otherwise in a credit line to the material. If material is not included in the article's Creative Commons license and your intended use is not permitted by statutory regulation or exceeds the permitted use, you will need to obtain permission directly from the copyright holder. To view a copy of this license, visit http://creativecommons.org/ licenses/by/4.0/

(C) The Author(s) 2019 